\title{
28 Research Square \\ LncRNA LINC00665 Promotes Ovarian Cancer Cell Proliferation and Inhibits Apoptosis via Targeting MiR-181a-5p/FHDC
}

\section{Suli Wang}

Nanjing Medical University Affiliated Healthcare Hospital for Women and Infants: Nanjing Maternity and Child Health Care Hospital

\section{Yingchun Wang}

Nanjing Medical University Affiliated Healthcare Hospital for Women and Infants: Nanjing Maternity and Child Health Care Hospital

Jin Lu Jiangsu Cancer Hospital

Jinhua Wang ( $\nabla$ drwangjinhua@tom.com )

Jiangsu Institute of Cancer Research: Jiangsu Cancer Hospital

\section{Research Article}

Keywords: LINC00665, miR-181a-5p, FHDC1, human ovarian cancer

Posted Date: October 14th, 2021

DOI: https://doi.org/10.21203/rs.3.rs-389237/v2

License: (c) (1) This work is licensed under a Creative Commons Attribution 4.0 International License. Read Full License 


\section{Abstract}

Objective: Previous reports indicate that long intergenic non-coding RNA LINC00665 naturally occurred vital effect in various cancers. Herein, the role of LINC00665 in ovarian cancer progress was explored.

Methods: We found that LINC00665 was upregulated in ovarian cancer cell lines. Besides that, A series of assays including flow cytometry, wound-healing, Transwell, Cell Counting Kit-8 (CCK-8), and EdU assay confirmed that the knockdown of LINC00665 could reduce the viability, proliferation and migration of SKOV-3 and OVCAR-3 cells. Accumulating evidence indicates that many IncRNAs can function as endogenous miRNA sponges by competitively binding common miRNAs. In this study, the bioinformatics analysis suggested that LNC00665 specifically binds to miR-181a-5p.

Results: LINC00665 downregulated the miR-181a-5p in SKOV-3 and OVCAR-3 cells. The knockdown of miR-181a-5p evidently reverses the inhibitory effect of sh-LINC00662. Besides, FH2 Domain Containing 1 (FHDC1) has been proved to deed as an effective target of miR-181a-5p.

Conclusion: Our results reveal the knockdown of LINC00665 facilitates ovarian cancer via development by sponging miR-181a-5p and upregulating FHDC1 expression; these may contribute to ovarian cancer therapy.

\section{Introduction}

Ovarian cancer (OC) is one of the highest lethal tumors all over the world (2). In the United States, there are about 22,000 new cases diagnosed and 14,000 deaths related to ovarian cancer in 2018 (22). In China, there are about 25,000 ovarian cancer deaths cases in 2015 (25). Although surgery, chemotherapy and radiotherapy serve significant progress in the treatment of $\mathrm{OC}$, patients with advanced $\mathrm{OC}$ with distant metastasis or recurrence remain incurable, and the median overall survival (OS) time of patients with metastatic disease has not improved in the past decades (14). It is urgently needed to develop an effective strategy to improve the early detection of OC. However, despite increasing reports on the molecular mechanisms of OC development, still little be known about the exact molecular mechanisms of ovarian cancer pathogenesis.

Long non-coding RNAs (IncRNAs) are the naturally occurring long intergenic non-coding RNA without coding proteins (17). During the past few decades, a great number of reports have disclosed that IncRNAs can act as scaffolds or sponges to modulate gene expression (9), which allows them to play some vital roles in cell proliferation, differentiation or apoptosis $(7,24)$. Numerous studies provided clear evidence that the aberrant IncRNAs play oncogenic or tumor-suppressive roles in ovarian cancer $(21,12)$. The dysregulation of IncRNAs was expected to become a potential prognostic and diagnostic biomarker, as well as a therapeutic target for ovarian cancer treatment. LINC00665 was first identified in oral premalignant lesions (8). After that, LINC00665 was observed in several other tumors, including Hepatocellular Carcinoma (HCC) (23) and lung adenocarcinoma (5). 
As LINC00665 was reported to promote several kinds of human tumors progression, but its involvement in ovarian cancer remained unknown. In the present study, the important role of LINC00665 was clearly elucidated as an oncogene to promote ovarian cancer progression. First, we showed the function of LINC00665 in ovarian cancer cells. Then, a series of experiments were carried out to demonstrate that LINC00665 upregulated FHDC1 expression by sponging miR-181a-5p. All of our results indicate that LINC00665 might take part in the inactivation of the miR-181a-5p-FHDC1 pathway; these may contribute to novel ovarian cancer therapy.

\section{Materials And Methods Cell culture}

Human ovarian epithelial cell line (ISOE80) and human ovarian cancer cell lines (SKOV-3, A2780, OVCAR3 and H08910) were purchased from American Type Culture Collection (ATCC, Manassas, VA, USA). After resuscitation, ISOE80 was cultured in a DMEM medium, while other cells were cultured in RPMI-1640 medium containing $10 \%$ fetal bovine serum (FBS, Beyotime, Beijing, China) in a humidified environment. When cells attained $70 \%$ to $80 \%$ confluence, transfection experiments could be conducted.

\section{Cell transfection}

The LINC00665-targeting shRNA sequences were designed and inserted into pENTR/U6 plasmids to construct LINC00665 shRNA. The NC mimic and miR-181a-5p mimic were purchased from Ribobio corporation (Guangzhou, China). Cell transfection was performed by using cell electroporation system operator H1 (Suzhou Etta Biotech Co. Ltd., Suzhou, China.) according to the manufacturer's manual. Cell transfection efficiency was measured by qRT-PCR at 48 hours post-transfection.

\section{CCK8 assay}

After cell transfection, SKOV-3 and OVCAR-3 were seeded into 96 -well plates at $5 \times 10^{3}$ per well. After incubated for 0 hours, 24 hours, 48 hours and 72 hours, $10 \mu \mathrm{L}$ CCK 8 solution were added and incubated for 2 hours in the dark. Then, absorbance at $450 \mathrm{~nm}$ was determined using a VarioskanTM LUX microplate reader (Thermo Fisher Scientific).

\section{EdU assay}

EdU assay was performed as described (1). Briefly, after electroporation treatment, $5 \times 10^{3} \mathrm{SKOV}-3$ and OVCAR-3 cells were seeded into each well of the 96-well plates and cultured for another 72 hours. Then, the cells were incubated with $10 \mu \mathrm{M}$ EdU for 4 hours and stained DAPI. Finally, the EdU-positive cells were 
analyzed by fluorescence microscope (Leica, Hilden, Germany), 200x, and densitometric analyzed the percentage using ImageJ (Bethesda, MD, USA).

\section{Cell cycle and cell apoptosis}

After cell electroporation, SKOV-3 and OVCAR-3 cells were seeded into 6 -well plates at $5 \times 10^{5}$ per well and cultured cells for the next 72 hours. Then, the tumor cells were harvested for cell cycle and apoptosis analysis.

For cell cycle analysis, the cells were incubated in ice-cold ethanol for 2 hours and then treated with RNase A ( $0.2 \mathrm{mg} / \mathrm{mL}$, Sigma-Aldrich). Next, the cells were incubated with propidium iodide $(2 \mu \mathrm{L})$ at room temperature for 40 minutes, and finally, cell cycle detection was analyzed.

For analysis, the tumor cells with indicated treatments were washed once with PBS. Then, the cells were resuspended in $100 \mu \mathrm{L}$ binding buffer and incubated with $10 \mu \mathrm{g} / \mathrm{mL}$ Annexin V-fluorescein isothiocyanate and $10 \mu \mathrm{g} / \mathrm{mL} \mathrm{PI} \mathrm{(both} \mathrm{Sigma-Aldrich)} \mathrm{at} \mathrm{room} \mathrm{temperature} \mathrm{for} 30$ minutes. Apoptosis analysis was performed using a FACScan flow cytometer (Becton Dickinson).

\section{Wound healing assay}

To study the migration of SKOV-3 and OVCAR-3 cells transfected with different reagents. The woundhealing assay was performed as described (18). Briefly, $5 \times 10^{5}$ transfected SKOV-3 or OVCAR-3 cells were seeded into each well in a 6-well plate. When the cells were cultured to $90 \%$ confluence, a wound was made by a $100 \mu \mathrm{l}$ size pipette tip. After another 48 hours, the wound recovery area was evaluated under a light microscope.

\section{Transwell assay}

To detect the capacity of migration, the Transwell assay was performed as described (13). Briefly, $3 \times 10^{4}$ transfected SKOV-3 or OVCAR-3 cells were seeded into the upper chamber of Matrigel-precoated transwell and cultured in RPMI-1640 medium. The lower chamber contained 10\% FBS growth medium. After 48 hours of culture, the transwell cells present in the lower chamber were fixed and stained with crystal violet. The pictures were taken under a light microscope, and individual cell colonies were counted.

\section{Luciferase reporter assay}

The Dual-Luciferase system was generated by inserting the cDNA fragments containing the putative miR181a-5p binding site from LINC00665 or FHDC1 3'-UTR into pmirGLO Dual-Luciferase miRNA Target Expression Vectors (Promega, Madison, WI, USA). PmirGLO/LINC00665 or pmirGLO/ FHDC1 3'-UTR 
constructs along with miR-181a-5p mimics were co-transfected into SKOV-3 and OVCAR-3 cells; then the cells were seeded into a 24-well plate for about 48 hours culture. Finally, the cells were lysed to measure the Dual-Luciferase Reporters' luciferase activity according to the manufacturer's protocol.

\section{Immunofluorescence staining}

After cell transfection, SKOV-3 and OVCAR-3 cells were seeded in 96-well plates at $5 \times 10^{3}$ per well and cultured for the next 72 hours. Then, the cells were fixed in $4 \%$ paraformaldehyde and permeabilized with xylene. Blocked with 5\% BSA in PBS, the cells were immunolabeled with primary antibody: FHDC (1:100; Proteintech) and then incubated with FITC-conjugated secondary antibody after washing by $\mathrm{xxx}$. The nuclei were counterstained using DAPI (Invitrogen), and the cells were observed under a fluorescence microscope (Olympus, Japan).

\section{RT-qPCR analysis}

TRIpure reagent (Invitrogen, USA) was used to isolate the total RNA from the cultured cells. PrimeScript RT kit (TaKaRa, Otsu, Japan) was used for reverse transcription. After the sample was prepared, the expression level was detected with SYBR green, and GAPDH was controlled as the internal parameter. Fold changes of gene experiments were measured by $2^{-\Delta \Delta C t}$ methods. Primers of LINC00665, miR-181a5p, U6, FHDC1 and GAPDH were as described in previous studies: LINC00665 sense, 5'AGCACCCCTAGTGTCAGTCA-3' and antisense, 5'-TGGTCTCTAGGGAGGCAGAA-3'; miR-181a-5p sense, 5'AACATTCAACGCTGTCGGTGAGT-3' and antisense, 5'-GTGCAGGGTCCGAGGT-3'; U6 sense, 5'CTCGCTTCGGCAGCACA-3' and antisense, 5'-AACGCTTCACGAATTTGCGT-3'; FHDC1 sense, 5'ACATCCAGCGGGATGGTGAACT-3' and antisense, 5'-GGAGCTCTTGTTTCCAGCATTCC-3'.

\section{Western blot}

The cultured cells were harvested in lysis buffer and incubated on ice for $30 \mathrm{~min}$. The supernatant was collected by centrifugation with $15,000 \mathrm{~g}$ for $5 \mathrm{~min}$ at $4{ }^{\circ} \mathrm{C}$. Then the proteins $(40 \mu \mathrm{g})$ were separated on $12 \%$ SDS-PAGE and transferred onto PVDF membranes (Millipore) using a MiniGenie blotting system (Bio-Rad). Next, the membranes were blocked for 1 hour with TBST containing 1\% skimmed milk powder and then incubated overnight at $4{ }^{\circ} \mathrm{C}$ with rabbit antibodies against human FHDC1 (1:1000; cat. no. NBP1-93579; Novus Biologicals), MMP2 (1:1000; cat. no. 40994S; Cell Signaling Technology, Danvers, MA, USA), MMP9 (1:1000; cat. no. 13667S; Cell Signaling Technology), Cyclin D1 (1:1000; cat. no. 55506S; Cell Signaling Technology), p21 (1:1000; cat. no. 2947S; Cell Signaling Technology), Bcl-2 (1:1000; cat. no. ab185002; Abcam), Bax (1:500; cat. no. ab53154; Abcam), Cleaved Caspase-3 (1:500; cat. no. ab49822; Abcam), Cleaved Caspase-9 (1:1000; cat. no. 20750S; Cell Signaling Technology) or GAPDH (1:1000; cat. no. 5174S; Cell Signaling Technology). Next, the membranes were washed with TBST and incubated with goat-anti-rabbit secondary antibody (1:10000; cat. no. 14708S; Cell Signaling 
Technology). Finally, the protein bands on the membranes were visualized with an enhanced chemiluminescence (ECL) system. Densitometric analysis of the bands was done using The ImageJ.

\section{Statistical Analysis}

All experiments were done in triplicates. GraphPad Prism (Version 6.01 for Windows) statistical software was used to perform statistical analysis. Student t-tests were employed to identify the significant differences between groups. One-way ANOVA and Tukey test were used to identify differences among three or more groups. Statistical significance difference was set at $p<0.05$.

\section{Results}

\section{LINC00665 is upregulated in ovarian cancer cell lines}

First of all, to confirm whether LINC00665 takes part in ovarian cancer, we employed RT-qPCR to compare its expression level in different human ovarian cancer cell lines (SKOV-3, A2780, OVCAR-3 and H08910) and human ovarian epithelial cell line (ISOE80). The results demonstrated that LINC00665 level was significantly upregulated in SKOV-3, A2780, OVCAR-3 and H08910 cell lines compared with the control ISOE80 cell line (Fig. 1A). As the expression level of LINC00665 in SKOV-3 and OVCAR-3 was highest among the human ovarian cancer cell lines, we chose SKOV-3 and OVCAR-3 as cell models to explore the biological function of LINC00665 in vitro.

\section{Knockdown of LINC00665 inhibits ovarian cancer cell proliferation and promotes the apoptosis}

To further explore the role of LINC00665 in the progression of ovarian cancer, we transfected sh-RNA targeting LINC00665 (sh-LINC00665) into SKOV-3 and OVCAR-3 cells. RT-qPCR showed that shLINC00665 significantly downregulated the expression of LINC00665 in SKOV-3 and OVCAR-3 cells (Fig. $1 \mathrm{~B}, p<0.01)$. CCK-8 assay showed that sh-LINC00665 remarkably reduced the viability of SKOV-3 and OVCAR-3 cells as compared to negative control (sh-NC) (Fig. 1C, $p<0.01$ ). While, EdU assay showed that sh-LINC00665 remarkably reduced the EdU positive cells compared to negative control (sh-NC) in both SKOV-3 and OVCAR-3 cells (Fig. 1D, $p<0.01$ ). Next, the data from flow cytometry assay indicated that shLINC00665 prominently increased the percentage of cells at the G0/G1 $(p<0.01)$, and decreased the percentage of $\mathrm{S}$ and G2/M phase in SKOV-3 and OVCAR-3 cells (Fig. 1E, $p<0.05$ ).

Meanwhile, the result of western blot showed that sh-LINC00665 remarkably reduced the expression of Cyclin D1 and increased the expression of p21 in SKOV-3 and OVCAR-3 cells (Fig. 1F, $p<0.01$ ). Besides that, flow cytometry analysis showed that knocking down LINC00665 observably facilitated ovarian cancer cell apoptosis (Fig. 1G, p<0.01). Additionally, western blot analysis showed that sh-LINC00665 
upregulates the protein levels of Bax, Cleaved Caspase- 3 and Cleaved Caspase-9, and downregulates the protein level of Bcl-2 in SKOV-3 and OVCAR-3 cell (Fig. 1H, $p<0.01)$.

\section{Knockdown of LINC00665 inhibits ovarian cancer cell migration and invasion}

Subsequently, the effect of LINC00665 on cell invasion and migration was examined using wound healing assay and transwell assay. The wound-healing assay showed that the reduced level of LINC00665 remarkably repressed the migratory ability of SKOV-3 and OVCAR-3 cells compared to the control (Fig. 2A, $p<0.01$ ). The transwell assay showed that sh-LINC00665 markedly inhibited the invasion of SKOV-3 and OVCAR-3 cells compared to sh-NC (Fig. 2B, $p<0.01$ ). Additionally, western blot was explored to measure the cell migration-related proteins. The result showed that sh-LINC00665 remarkably downregulates the expression levels of MMP-2 and MMP-9 in SKOV-3 and OVCAR-3 cells compared to shNC (Fig. 2C, $p<0.01$ ). Taken together, these findings demonstrate that LINC00665 promotes ovarian cancer cells proliferation, migration and invasion and inhibits cell apoptosis in vitro.

\section{MiR-181a-5p is the target gene of LINC00665}

Since one-way IncRNAs regulate gene expression is to act as a miRNA sponge, we predicted the potential miRNAs targets of LINC00665 in DIANA-LncBase (https://bigd.big.ac.cn). The result showed one of the potential targets was miR-181a-5p (Fig. 3A). To further reveal the relationship between LINC00665 and miR-181a-5p. We constructed miR-181a-5p mimic to overexpress miR-181a-5p in SKOV-3 and OVCAR-3 cells. RT-qPCR showed that miR-181a-5p mimic significantly upregulated the expression of miR-181a-5p in SKOV-3 and OVCAR-3 cells (Fig. 3B, $p<0.01$ ). Then, dual-luciferase reporter assay was performed to verify this relationship, and the results indicated that miR-181a-5p mimic significantly downregulated the luciferase activity of the LINC00665-WT reporter vector (Fig. 3C). The downregulation of the luciferase activity of LINC00665 reporter vector was abrogated when the predicted binding site was mutated (Fig. 3C). Moreover, our results show that LINC00665 knockdown upregulated miR-181a-5p expression in SKOV-3 and OVCAR-3 cells (Fig. 3D). In addition, the result of RT-qPCR demonstrated that the expression level of miR-181a-5p was significantly lower in SKOV-3, A2780, OVCAR-3 and H08910 cell lines compared with the control ISOE80 cell line (Fig. 3E). Overall, these results demonstrated that LINC00665 directly interacted with miR-181a-5p and influenced its expression in ovarian cancer cells.

\section{MiR-181a-5p regulates FHDC1 expression in SKOV-3 and OVCAR-3 cells}

We next explored the target genes of miR-181a-5p. Bioinformatics analysis via 
Targetscan (http://www.targetscan.org) showed that one of the target genes of miR-181a-5p was FHDC1 (Fig. 4A). We performed a dual-luciferase reporter assay to verify this interaction. The result indicated that the overexpression of miR-181a-5p significantly downregulated FHDC1-WT reporter vector's luciferase activity (Fig. 4B, $p<0.01$ ). Meanwhile, overexpression miR-181a-5p with a mutation within the predicted miR-181a-5p binding site did not influence luciferase activity of the FHDC1 reporter vector (Fig. 4B). We determined both the RNA and protein expression level of FHDC1 in the SKOV-3 and OVCAR-3 cells overexpressed miR-181a-5p with RT-qPCR, western blot and immunofluorescence. The results demonstrated that FHDC1 was significantly downregulated in the SKOV-3 and OVCAR-3 cells overexpressed miR-181a-5p compared to the negative control (Fig. 4C, 4D and 4E). Similarly, the expression level of FHDC1 level was significantly upregulated in SKOV-3, A2780, OVCAR-3 and H08910 cell lines compared with the control ISOE80 cell line (Fig. 4F). These data confirmed that miR-181a-5p could regulate FHDC1 expression in ovarian cancer.

\section{miR-181a-5p/FHDC1 mediated the effect of LINC00665 knockdown on SKOV-3 and OVCAR-3 cells}

To confirm the interaction of miR-181a-5p, FHDC1 and LINC00665 in OC, we co-transfected SKOV-3 cells with sh-LINC00665, or sh-LINC00665 with miR-181a-5p inhibitor, or their combinations with sh-FHDC1. RT-qPCR results showed that after transfection of miR-181a-5p inhibitor, the expression of miR-181a-5p in cells was significantly down-regulated. However, in sh-LINC00665 transfected cells, FHDC1 was significantly down-regulated (Figure $5 \mathrm{~A}, \mathrm{p}<0.01$ ). CCK8 assay revealed that the cells transfected shLINC00665 and NC inhibitor showed lowest cell viability, while the viability of cells transfected shLINC00665 along with miR-181a-5p inhibitor and sh-FHDC1 markedly increased compared to the cells transfected sh-LINC00665 and miR-181a-5p (Fig. 5B, p<0.05). Besides, as shown in Fig. 5C and Fig. 5D, the cells transfected sh-LINC00665 and NC inhibitor showed the highest level of cell apoptosis. Downregulation of miR-181a-5p remarkably inhibited the cell apoptosis induced by shLINC00665. Meanwhile, sh-FHDC1 markedly reversed the inhibition effect of miR-181a-5p inhibitor on SKOV-3 cells apoptosis $(p<0.05)$. Furthermore, the wound healing assay delineated that downregulation of miR-181a-5p remarkably improved the migration of the SKOV-3 cells with LINC00665 knockdown (Fig. $5 \mathrm{E})$, compared to the cells co-transfected with sh-LINC00665 and NC inhibitor, while sh-FHDC1 markedly reversed the improvement from miR-181a-5p inhibitor (Fig. 5F, $p<0.05$ ). Similarly, the transwell assay delineated that the downregulation of miR-181a-5p markedly aggravated the invasion of SKOV-3 cells with LINC00665 knockdown. And sh-FHDC1 remarkably inhibited such a restoration effect (Fig. 5F, $p<0.05)$.

\section{Discussion}

Ovarian cancer is one of the most common and aggressive malignancies among women around the world. Reveal the molecular mechanisms of ovarian cancer pathogenesis, and find the potential diagnostic biomarkers and therapeutic targets may be one of the best ways to reduce the harm of ovarian 
cancer to women. In the present research, we demonstrated that LINC00665 was up-regulated in ovarian cancer cell lines. Knockdown of LINC00665 dramatically reduced the cell visibility, arrested the cell cycle at the G0/G1 phase, and inhibited the migration and invasion of SKOV-3 and OVCAR-3 cells in vitro. This preliminary research indicated that LINC00665 might be a promising candidate for ovarian cancer therapy.

Increasing evidence indicates that dysregulation of IncRNAs plays an important role in tumorigenesis and metastasis in human cancer (10). In ovarian cancer, several IncRNAs have been identified as indicators for patients' prognosis. For example, the expression of IncRNA ROR has been studied significantly higher in the plasma of ovarian cancer patients than that in healthy controls. And the enhanced level of plasma IncRNA ROR could be used as a potential biomarker for the diagnosis of ovarian cancer (19). By contrast, the expression of IncRNA CASC15 was lower in ovarian cancer tissues. And the Kaplan-Meier survival analysis showed that patients with low CASC15 expression levels had poorer overall survival and progression-free survival than those with high CASC15 expression (20). In the present research, we demonstrated that LINC00665 expression level was significantly higher in ovarian cancer cell lines SKOV3, A2780, OVCAR-3 and H08910) compared to the human ovarian epithelial cell line (ISOE80).

LINC00665 was first reported in oral premalignant lesions as potential molecular pathogenesis and biomarker of high-risk oral premalignant lesions [11]. Next, LINC00665 was observed upregulated in several other tumors, including hepatocellular carcinoma (23) and lung adenocarcinoma (5). These were consistent with our results. As analysis of the cancer genome atlas and the gene expression omnibus showed overexpression of LINC00665 in patients with HCC was significantly associated with gender, tumor grade, stage, and tumor cell type. Overexpression of LINC00665 in patients with HCC was significantly associated with overall survival (OS) (HR $=1.47795 \%$; $\mathrm{Cl}: 1.046-2.086)(23)$. Besides that, bioinformatics analysis identified ten identified core genes of LINC00665 that regulate pathways involved in the cell cycle. This indicated LINC00665 facilitates the development and progression of HCC(23). In gastric cancer, LINC00665 might serve as a ceRNA for miR-149-3p to regulate the expression of RNF2. And LINC00665 suppression significantly reduced GC cells viability and invasion ability in vitro(16). In Prostate Cancer, LINC00665 was the sponge for miR-1224-5p. LINC00665 knockdown markedly attenuated growth and metastasis of PC cells and impaired tumor propagation in vivo(4). Our study demonstrated that LINC00665 knockdown could inhibit the proliferation, migration and invasion of ovarian cancer cells (SKOV-3 and OVCAR-3).

The evidence above suggests that LINC00665 could play a role of miRNA sponges to downregulate the activity of target miRNAs in many cancers $(16,4)$. Here, bioinformatics analysis identified miR-181a-5p as the target miRNAs of LINC00665, as they have complementary binding sites. The binding sites of miR181a-5p were also found in the 3 '-UTR of FHDC1, and they were validated with the luciferase assay. A previous study has shown that miR-181a-5p was downregulated in aggressive human breast and colon cancers and indicated that miR-181a-5p could inhibit tumor cell growth and invasion by attenuation elevated MMP-14 expression(11). Most importantly, one hundred sixty-four matched tumor biopsies study has shown that miR-181a-5p was associated with overall and progression-free survival $(P<0.05)$, 
residual tumor volume and Pt-free interval inv ovarian cancer(15). As previous studies reported, endogenous FHDC1 regulates Golgi ribbon formation and has an apparent preferential association with the Golgi-derived microtubule network. Knockdown of FHDC1 expression results in defective Golgi assembly and suggests a role for FHDC1 in the maintenance of the Golgi-derived microtubule network(6). Besides that, FHDC1 was found to affect the cell cycle and cell division(3). We found that LINC00665 can downregulate the expression of miR-181a-5p via direct interaction and upregulate the expression of FHDC1 in SKOV-3 and OVCAR-3 cells. At the same time, we found that miR-181a-5p decreased the expression of FHDC1 and reversed the effect of LINC00665 knockdown in SKOV-3 cells. Meanwhile, knockdown FHDC1 could reversed the effect of miR-181a-5p inhibitor in SKOV-3 cells. All of these data indicated LINC00665 might take part in the inactivation of the miR-181a-5p-FHDC1 pathway.

In summary, the present study provides evidence that LINC00665 modulates miR-181a-5p/FHDC1 pathway to facilitate ovarian cancer progression. Our data suggest that LINC00665- miR-181a-5p FHDC1 signal axis may provide a new therapeutic target for ovarian cancer therapy.

\section{Declarations}

\section{Ethics approval and consent to participate}

Not applicable.

\section{Consent for publication}

Not applicable.

\section{Availability of data and materials}

All data generated or analysed during this study are included in this published article.

\section{Competing interests}

The authors state that there are no conflicts of interest.

\section{Funding}

Not applicable.

\section{Authors' contributions}


S.W conducted most of the experiments. J.W. interpreted and analyzed the data. Y.W wrote the first draft of the article. J.L. finalized the manuscript. J.W. conceived the study and revised the manuscript. All authors read and approved the final manuscript.

\section{Acknowledgements}

Not applicable.

\section{References}

1. Adami, G. R., Tangney, C. C., Tang, J. L., Zhou, Y., Ghaffari, S., Naqib, A. ... Schwartz, J. L. (2018). Effects of green tea on miRNA and microbiome of oral epithelium. Sci Rep, 8, 5873

2. Bray, F., Ferlay, J., Soerjomataram, I., Siegel, R. L., Torre, L. A., \& Jemal, A. (2018). Global cancer statistics 2018: GLOBOCAN estimates of incidence and mortality worldwide for 36 cancers in 185 countries. CA Cancer J Clin, 68, 394-424

3. Chen, H. Q., Zhao, J., Li, Y., He, L. X., Huang, Y. J., Shu, W. Q. ... Liu, J. Y. (2018). Gene expression network regulated by DNA methylation and microRNA during microcystin-leucine arginine induced malignant transformation in human hepatocyte L02 cells. Toxicol Lett, 289, 42-53

4. Chen, W., Yu, Z., Huang, W., Yang, Y., Wang, F., \& Huang, H. (2020). LncRNA LINC00665 Promotes Prostate Cancer Progression via miR-1224-5p/SND1 Axis. Onco Targets Ther, 13, 2527-2535

5. Cong, Z., Diao, Y., Xu, Y., Li, X., Jiang, Z., Shao, C. ... Qiang, Y. (2019). Long non-coding RNA linc00665 promotes lung adenocarcinoma progression and functions as ceRNA to regulate AKR1B10-ERK signaling by sponging miR-98. Cell Death Dis, 10, 84

6. Copeland, S. J., Thurston, S. F., \& Copeland, J. W. (2016). Actin- and microtubule-dependent regulation of Golgi morphology by FHDC1. Mol Biol Cell, 27, 260-276

7. DiStefano, J. K. (2018). The Emerging Role of Long Noncoding RNAs in Human Disease. Methods Mol Biol, 1706, 91-110

8. Jia, H., Wang, X., \& Sun, Z. (2018). Exploring the molecular pathogenesis and biomarkers of high risk oral premalignant lesions on the basis of long noncoding RNA expression profiling by serial analysis of gene expression. Eur J Cancer Prev, 27, 370-378

9. Lalevee, S., \& Feil, R. (2015). Long noncoding RNAs in human disease: emerging mechanisms and therapeutic strategies. Epigenomics, 7, 877-879

10. Li, Q., Zhang, J., Zhou, J., Yang, B., Liu, P., Cao, L. ... Liu, H. (2018). IncRNAs are novel biomarkers for differentiating between cisplatin-resistant and cisplatin-sensitive ovarian cancer. Oncol Lett, 15, $8363-8370$

11. Li, Y., Kuscu, C., Banach, A., Zhang, Q., Pulkoski-Gross, A., Kim, D. ... Cao, J. (2015). miR-181a-5p Inhibits Cancer Cell Migration and Angiogenesis via Downregulation of Matrix Metalloproteinase- 14 . Cancer Res, 75, 2674-2685 
12. Lv, P., Qiu, X., Gu, Y., Yang, X., Xu, X., \& Yang, Y. (2019). Long non-coding RNA SNHG6 enhances cell proliferation, migration and invasion by regulating miR-26a-5p/MAPK6 in breast cancer. Biomed Pharmacother, 110, 294-301

13. Marshall, J. (2011). Transwell((R)) invasion assays. Methods Mol Biol, 769, 97-110

14. Marth, C., Reimer, D., \& Zeimet, A. G. (2017). Front-line therapy of advanced epithelial ovarian cancer: standard treatment. Ann Oncol, 28(Suppl 8), viii36-viii39

15. Petrillo, M., Zannoni, G. F., Beltrame, L., Martinelli, E., DiFeo, A., Paracchini, L. ... Marchini, S. (2016). Identification of high-grade serous ovarian cancer miRNA species associated with survival and drug response in patients receiving neoadjuvant chemotherapy: a retrospective longitudinal analysis using matched tumor biopsies. Ann Oncol, 27, 625-634

16. Qi, H., Xiao, Z., \& Wang, Y. (2019). Long non-coding RNA LINC00665 gastric cancer tumorigenesis by regulation miR-149-3p/RNF2 axis. Onco Targets Ther, 12, 6981-6990

17. Qiu, M. T., Hu, J. W., Yin, R., \& Xu, L. (2013). Long noncoding RNA: an emerging paradigm of cancer research. Tumour Biol, 34, 613-620

18. Rodriguez, L. G., Wu, X., \& Guan, J. L. (2005). Wound-healing assay. Methods Mol Biol, 294, 23-29

19. Shen, W., Xie, X., Liu, M., \& Wang, L. (2020). Diagnostic Value of IncRNA ROR in Differentiating Ovarian Cancer Patients. Clin Lab, 66.

20. Shi, Y., Gao, S., Zheng, Y., Yao, M., \& Ruan, F. (2019). LncRNA CASC15 Functions As An Unfavorable Predictor Of Ovarian Cancer Prognosis And Inhibits Tumor Progression Through Regulation Of miR221/ARID1A Axis. Onco Targets Ther, 12, 8725-8736

21. Sun, X., Huang, T., Zhang, C., Zhang, S., Wang, Y., Zhang, Q., \& Liu, Z. (2019). Long non-coding RNA LINC00968 reduces cell proliferation and migration and angiogenesis in breast cancer through upregulation of PROX1 by reducing hsa-miR-423-5p. Cell Cycle, 18, 1908-1924

22. Torre, L. A., Trabert, B., DeSantis, C. E., Miller, K. D., Samimi, G., Runowicz, C. D. ... Siegel, R. L. (2018). Ovarian cancer statistics, 2018. CA Cancer J Clin, 68, 284-296

23. Wen, D. Y., Lin, P., Pang, Y. Y., Chen, G., He, Y., Dang, Y. W., \& Yang, H. (2018). Expression of the Long Intergenic Non-Protein Coding RNA 665 (LINC00665) Gene and the Cell Cycle in Hepatocellular Carcinoma Using The Cancer Genome Atlas, the Gene Expression Omnibus, and Quantitative RealTime Polymerase Chain Reaction. Med Sci Monit, 24, 2786-2808

24. Xue, M., Zhuo, Y., \& Shan, B. (2017). MicroRNAs, Long Noncoding RNAs, and Their Functions in Human Disease. Methods Mol Biol, 1617, 1-25

25. Zhang, M. L., Peng, P., Wu, C. X., Gong, Y. M., Zhang, S. W., Chen, W. Q., \& Bao, P. P. (2019). [Report of breast cancer incidence and mortality in China registry regions, 2008-2012]. Zhonghua Zhong Liu Za Zhi, 41, 315-320

\section{Figures}


A
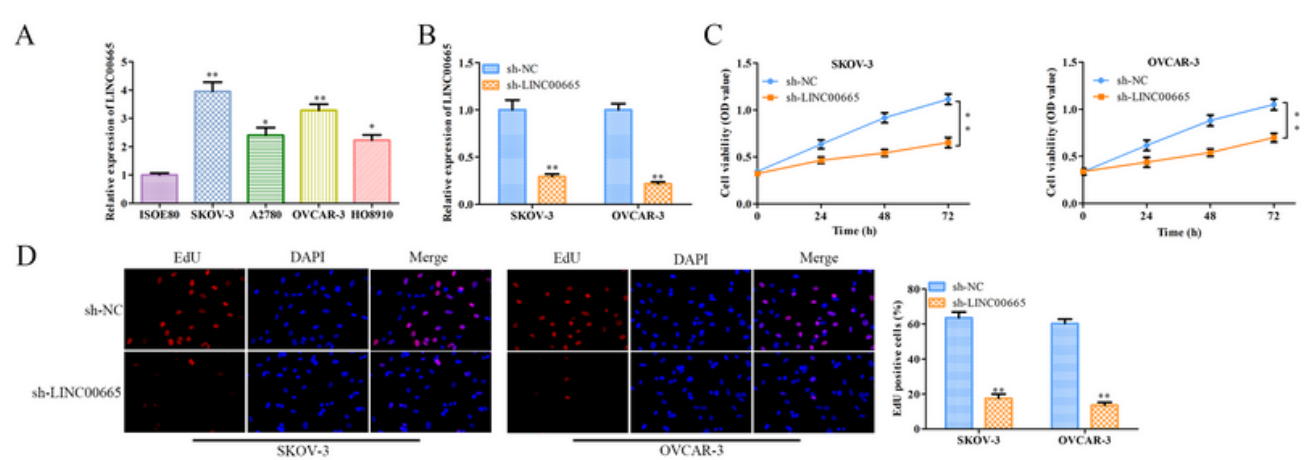

E
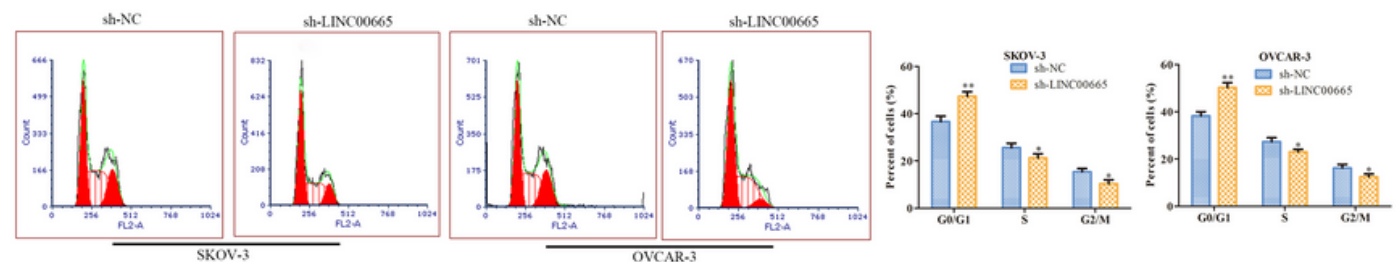

$\mathrm{F}$
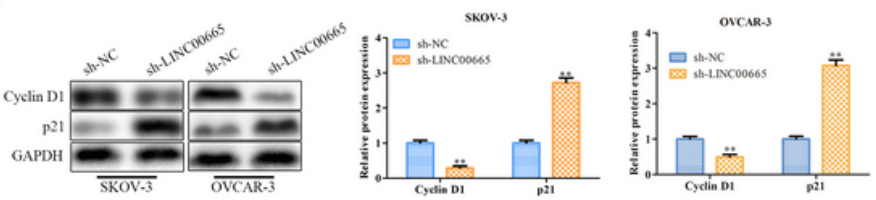

G
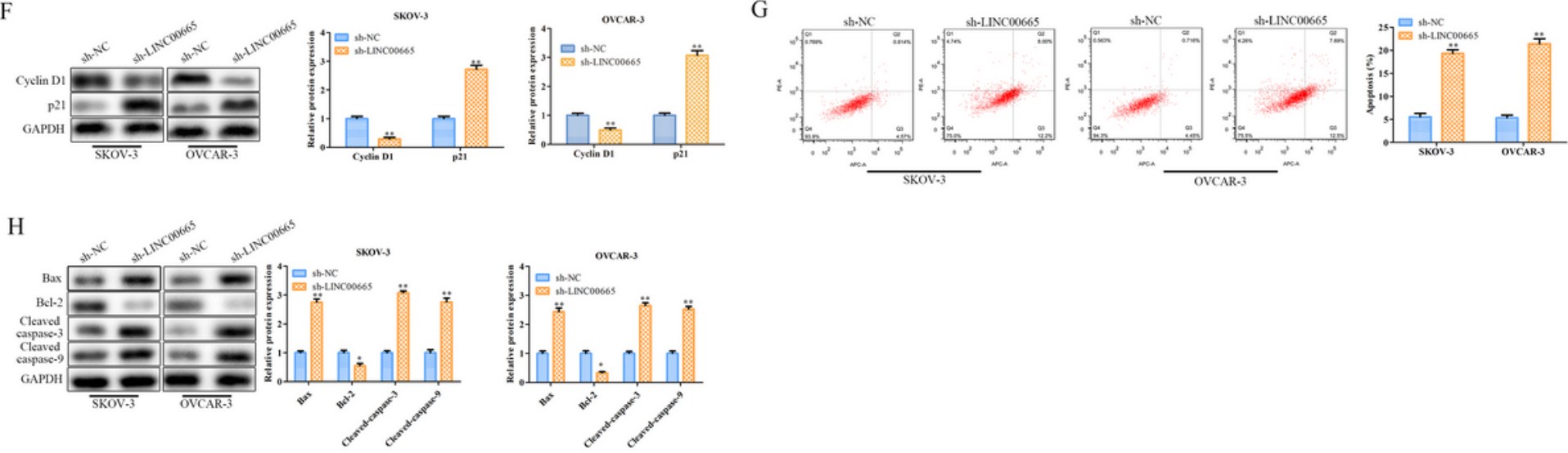

Figure 1

LINC00665 knockdown inhibits ovarian cancer cell proliferation and promotes apoptosis (A) RT-qPCR analysis was used to determine the relative expression of LINC00665 in ovarian cancer cells and ovarian epithelial cell. The results were normalized to GAPDH expression. (B) RT-qPCR was used to assess the expression of LINC00665 in SKOV-3 and OVCAR-3 cells transfected with sh-LINC00665 or sh-NC. (C) Cell viability of SKOV-3 and OVCAR-3 cell lines was measured employing CCK-8 assay.(D) Cell viability of SKOV-3 and OVCAR-3 cell lines was measured employing EdU assay.(E) Flow cytometry assay was performed to assess the cell cycle. (F) Western blot analysis was used to assess the expression of Cyclin and p21 proteins.(G) SKOV-3 and OVCAR-3 cell apoptosis ratio was detected by flow cytometry. $(\mathrm{H})$ Western blot analysis was used to assess the expression of cell apoptosis related proteins. Three independent experiments were performed. The graph shows the mean \pm SD calculated for at least three experiments. ${ }^{*} \mathrm{P}<0.05,{ }^{*} \mathrm{p}<0.01$. 
A
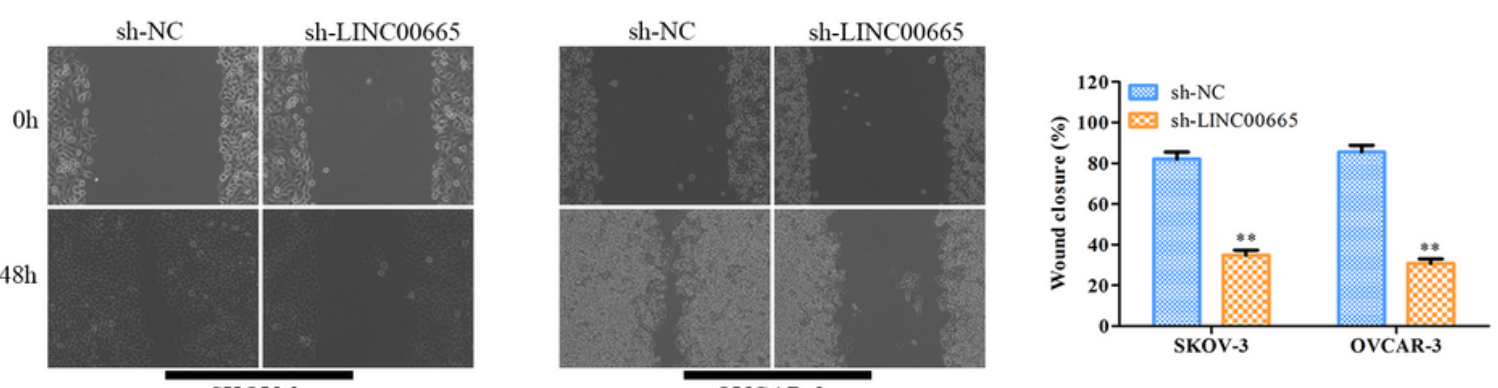

B
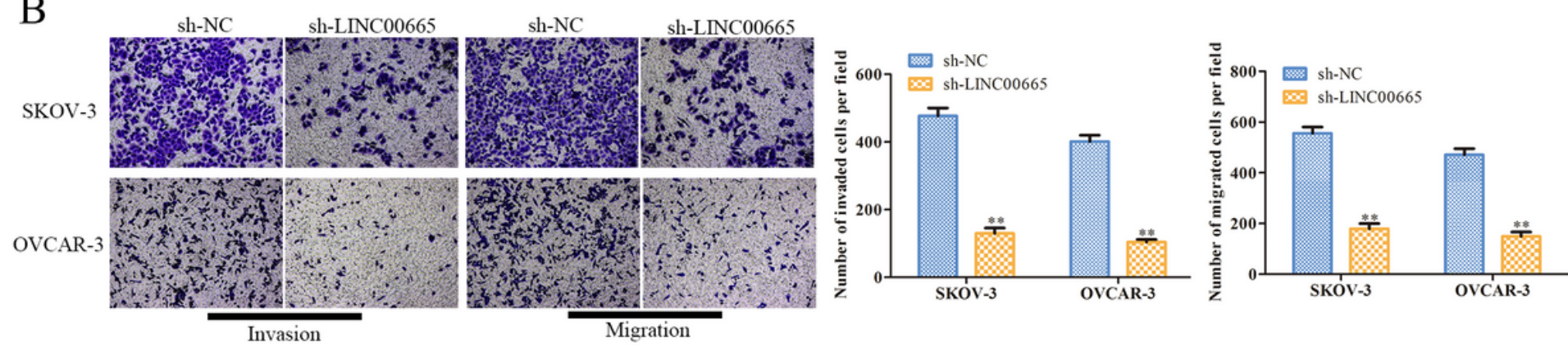

$\mathrm{C}$
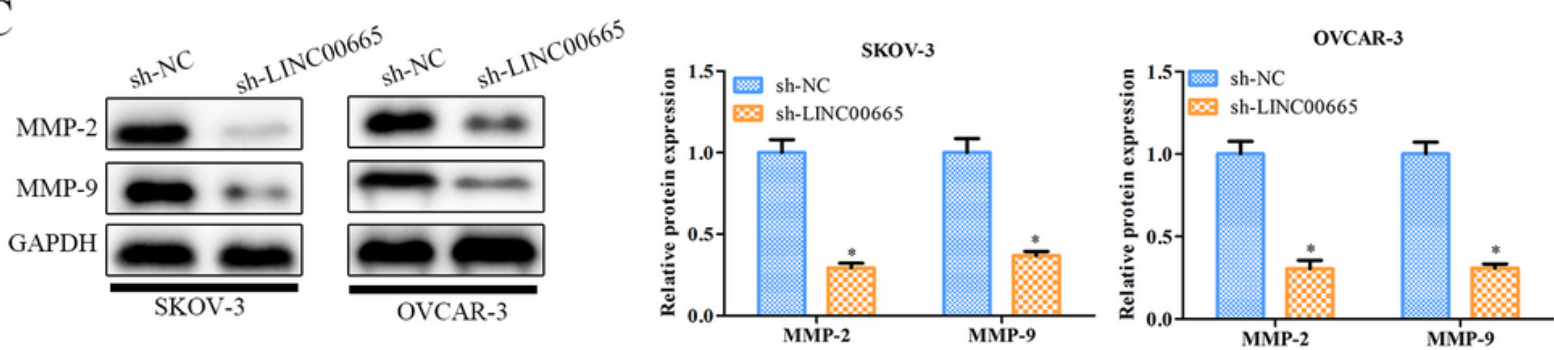

Figure 2

LINC00665 knockdown inhibits migration and invasion of SKOV-3 and OVCAR-3 cells. Wound healing assay (A), and transwell assay was (B), were performed to assess the migration and invasion of SKOV-3 and OVCAR-3 cells transfected with sh-LINC00665 or sh-NC.(C) Western blot analysis was used to assess the expression of MMP-2 and MMP-9 proteins. Three independent experiments were performed. The graphs show the mean $\pm S D$ of at least three experiments. ${ }^{*} p<0.05,{ }^{\star *} p<0.01$. 


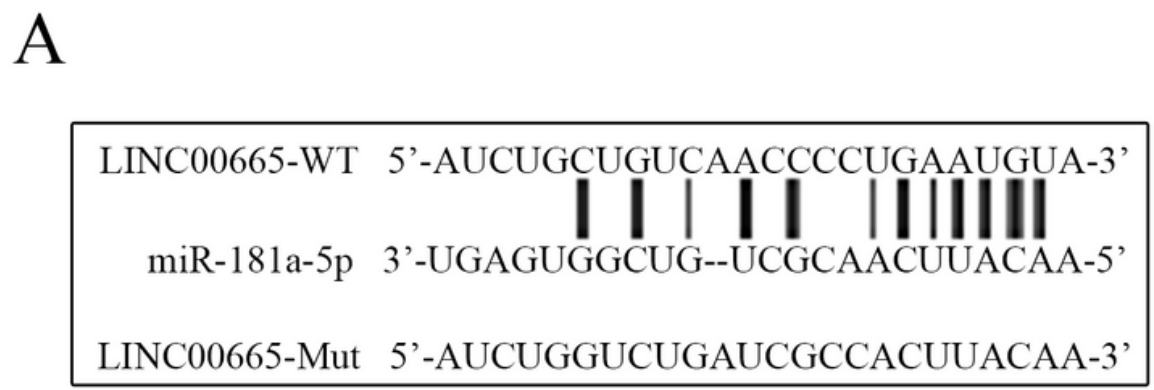

$\mathrm{C}$

SKOV-3

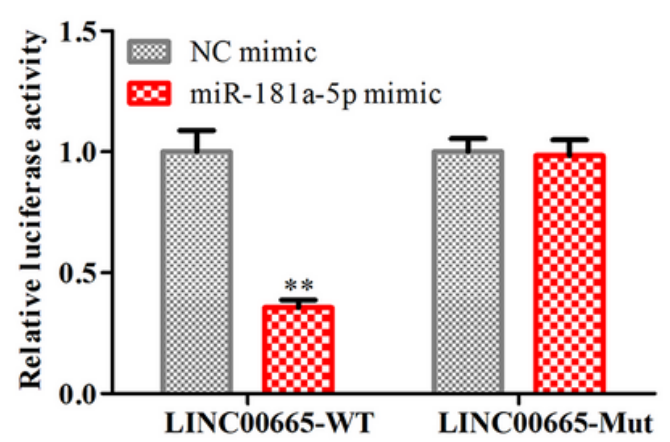

$\mathrm{D}$

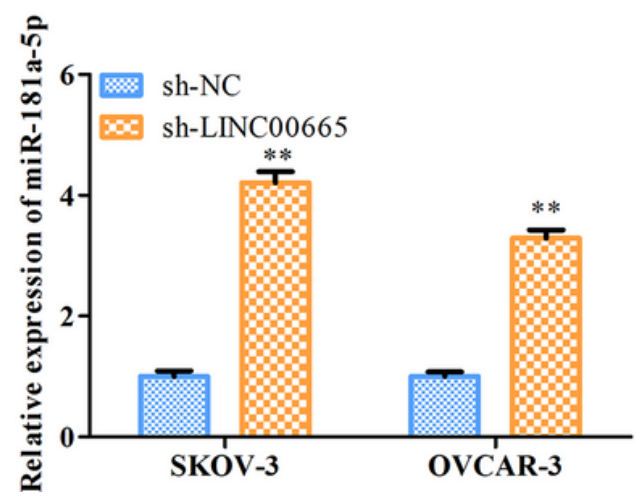

$\mathrm{B}$

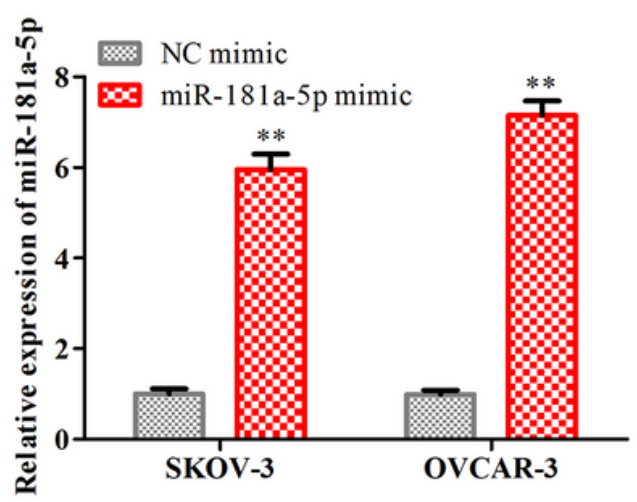

OVCAR-3

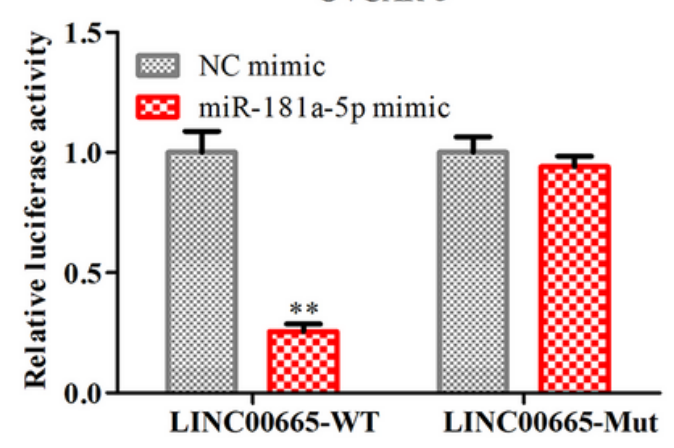

$\mathrm{E}$

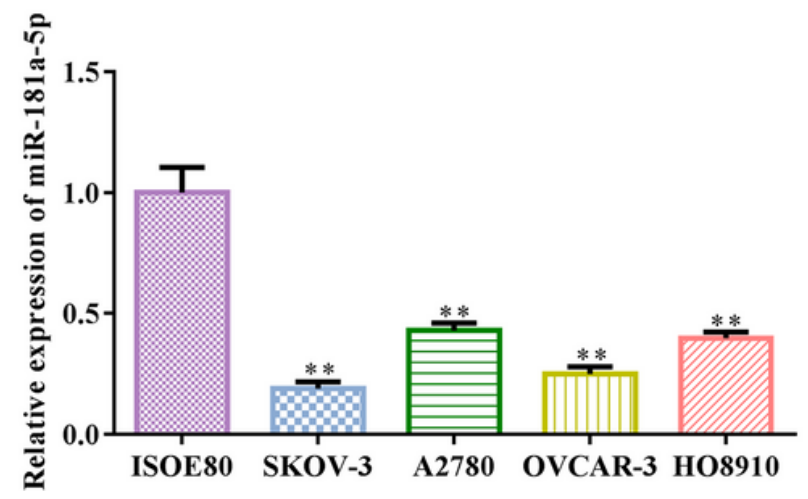

\section{Figure 3}

miR-181a-5p is a direct target of LINC00665. (A)DIANALncBase was employed to predict the target microRNA regulated by LINC00665. Binding region between miR-181a5p and LINC00665 was predicted, and LINC00665-Mut sequences were constructed. (B) RT-qPCR was taken to assess the expression of miR-181a-5p in OVCAR-3 and SKOV-3 cells transfected with miR-181a-5p mimic or NC mimic.(C) The luciferase reporter constructs containing the wild type (LINC00665-WT) or mutant LINC00665 (LINC00665-Mut) sequences were co-transfected into OVCAR-3 and SKOV-3 cells with miR-181a-5p mimic or NC mimic, followed by detecting of the relative luciferase activity.RT-qPCR was taken to assess the expression of miR-181a-5p in OVCAR-3 and SKOV-3 cells transfected with sh-LINC00665 or sh-NC. (D) RT-qPCR analysis was used to determine the expression of miR-181a-5p in ovarian cancer cells and ovarian epithelial cells. Three independent experiments were performed. The graph showed the mean \pm SD calculated for at least three experiments. ${ }^{*} p<0.05,{ }^{* \star} p<0.01$. 


$\begin{array}{lc}\text { FHDC1 WT } & 5 \text { '-gaaauugcuuucagaaGAAUGUa-3' } \\ \text { Hsa-miR-181a-5p } & 3 \text { '-ugaguggcugucgcaaCUUACAa-5 ' } \\ \text { FHDC1 Mut } & 5 \text { '-gaaauugcuuucagaaCUUACAa-3' }\end{array}$

$\mathrm{C}$

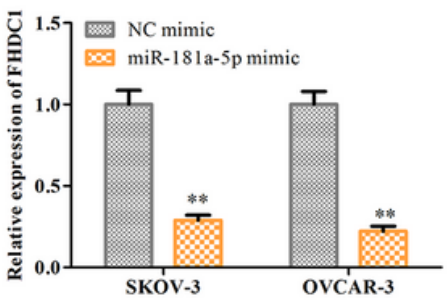

$\mathrm{E}$

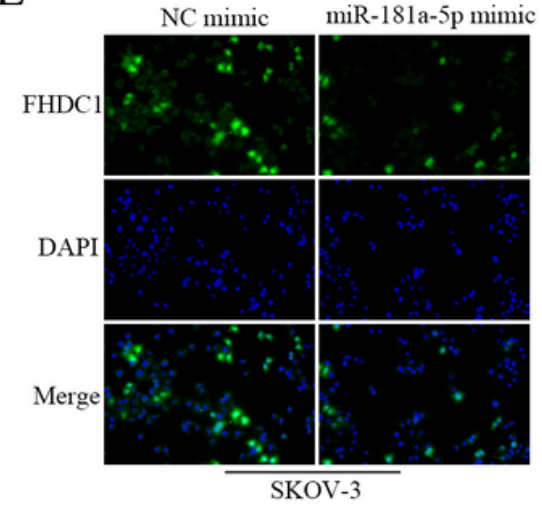

$\mathrm{D}$
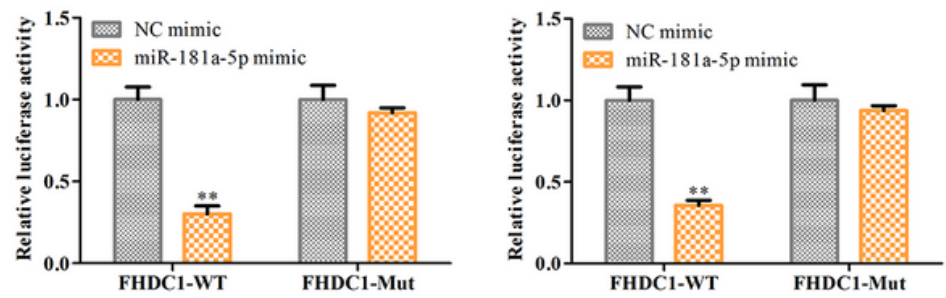

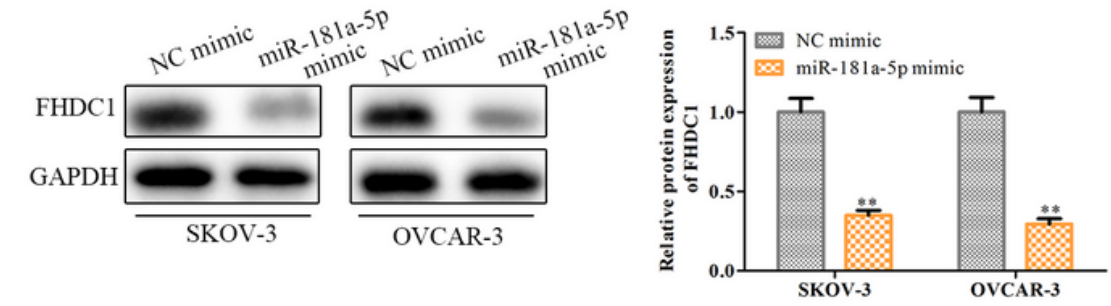

$\mathrm{F}$
NC mimic miR-181a-5p mimic

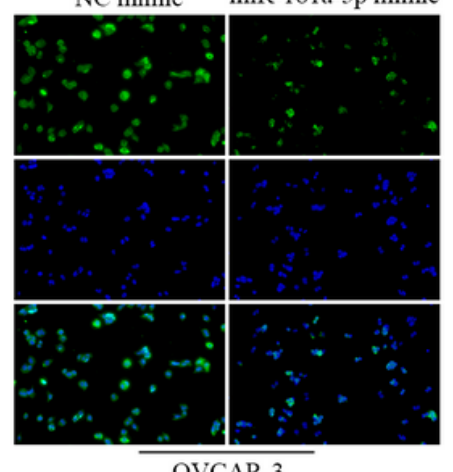

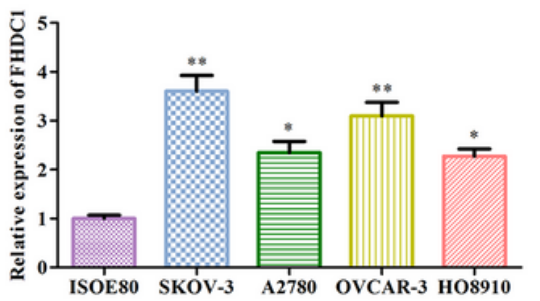

Figure 4

FHDC1 is a target gene of miR-181a-5p. (A) Targetscan was employed to predict the target genes regulated by miR-181a-5p. Binding region between miR-181a-5p and FHDC1 was predicted, and an FHDC1-Mut sequence was designed. (B) The luciferase reporter constructs containing the wild type (FHDC1-WT) or mutant FHDC1 (FHDC1-Mut) sequences were co-transfected into OVCAR-3 and SKOV-3 cells with miR-181a-5p mimic or the corresponding negative control (NC mimic), followed by the detection of the relative luciferase activity.RT-qPCR analysis (C), western blot (D) and immunofluorescence (E) were used to detect the expression level of FHDC1 in in OVCAR-3 and SKOV-3 cells transfected with miR-181a$5 p$ mimic or NC mimic.(F) RT-qPCR analysis was used to detect the expression level of FHDC1 in ovarian cancer cells and ovarian epithelial cells. Three independent experiments were performed. The graph showed the mean \pm SD calculated for at least three experiments. ${ }^{*} p<0.05,{ }^{* *} p<0.01$. 
A

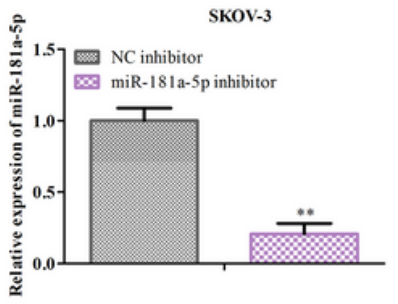

SKOV-3

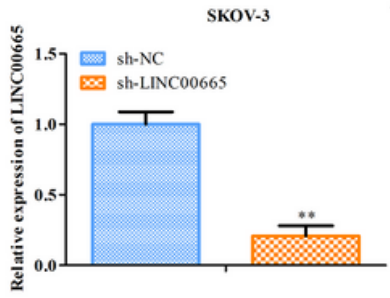

B

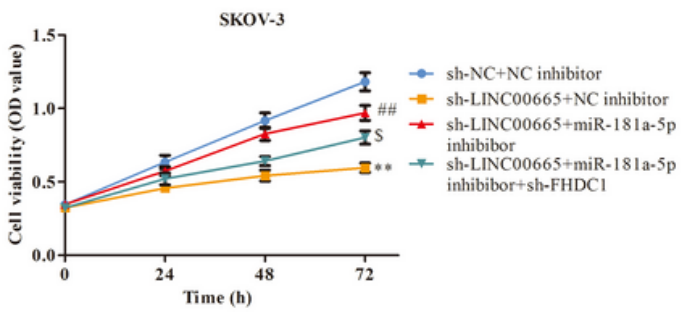

C
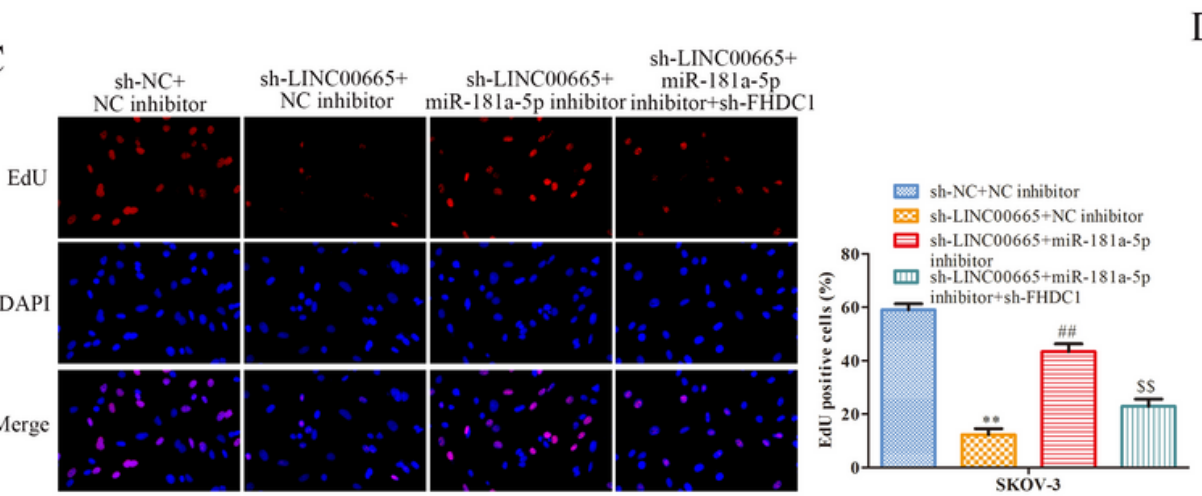

D

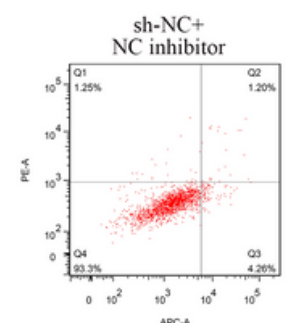

nPCA

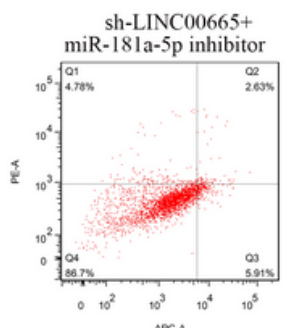

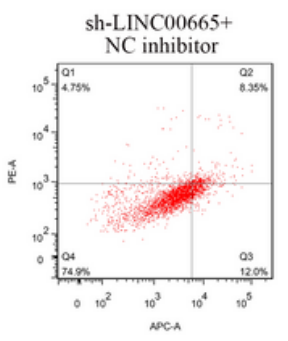

sh-LINC00665+ miR-181a-5p inhibitor+sh-FHDC

E
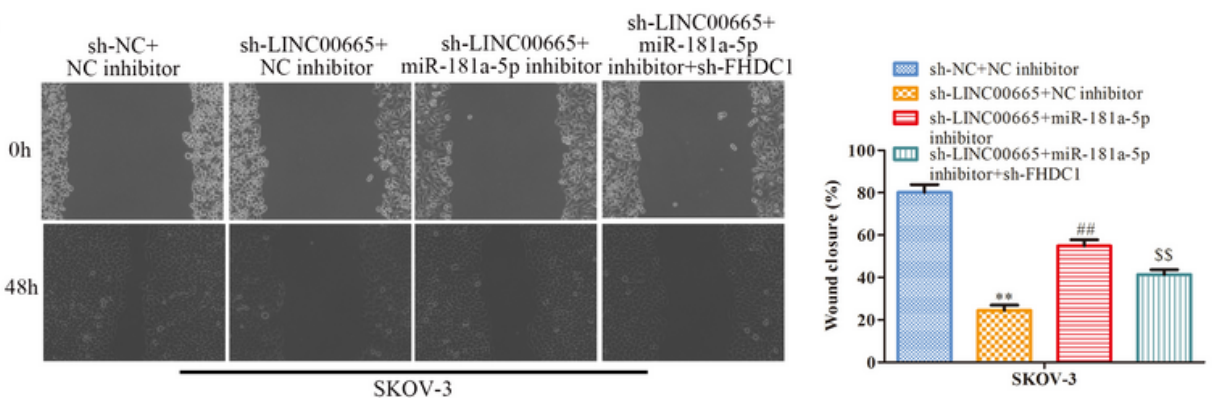

F
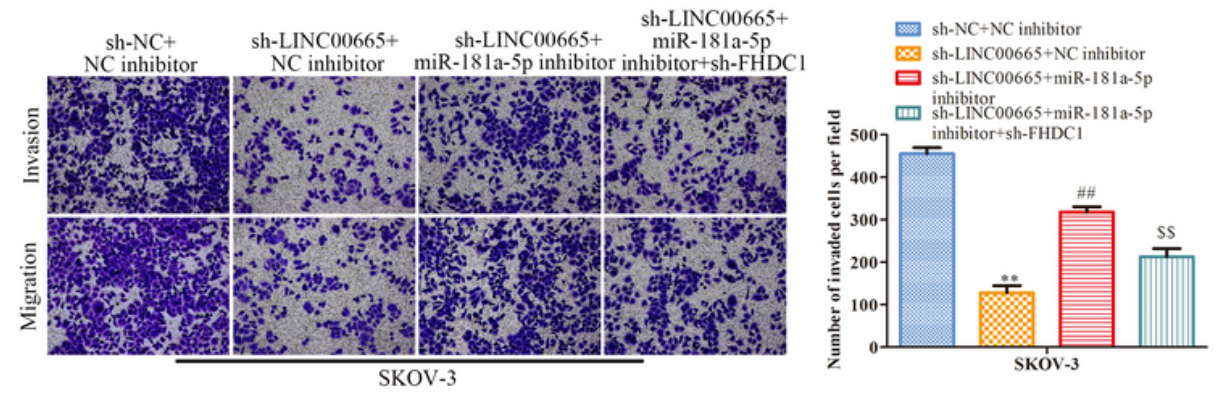

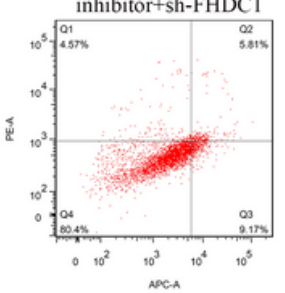

sh-NC+NC inhibitor

sh-LINC00665+NC inhibitor

曰 sh-LINC00665+miR-181a-5p

孟 inhibitor sh-LINC00665+miR-181a-5p

$\left.{ }^{25}\right] \quad$ inhibitor+sh-FHD
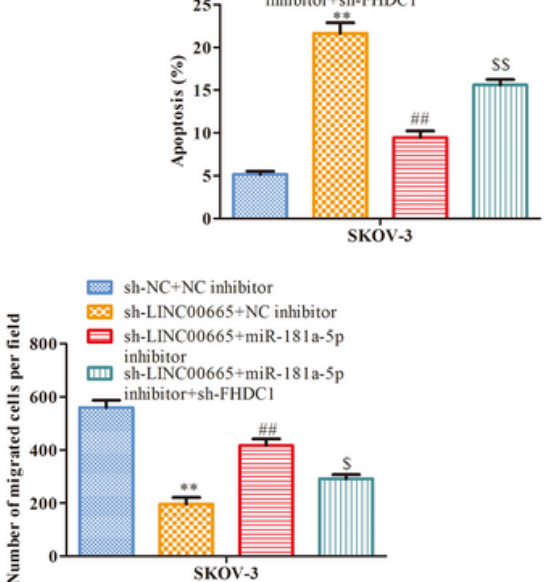

Figure 5

Compensation experiment reveals the relationship among FHDC1, miR-181a-5p and LINC00665 in SKOV3 cells. Sh-LINC00665 or sh-NC along with miR-181a-5p inhibitor and sh- FHDC1 were co-transfected into SKOV-3 cells. After transfection, the cells were seeded into appropriate cell culture plates for further assays. (A) RT-qPCR was used to assess the expression of miR-181a-5p in SKOV-3 cells with miR-181a$5 p$ inhibitor or the corresponding negative control (NC inhibitor). (B) RT-qPCR was used to assess the expression of FHDC1 in SKOV-3 cells with sh-LINC00665 or sh-NC.Cell viability of SKOV-3 cell lines was measured employing CCK-8 assay (C) and EdU assay (D). Wound healing assay (E), and transwell assay was (F), were performed to assess the migration and invasion of SKOV-3 cells. Three independent 
experiments were performed. The graph showed the mean \pm SD calculated for at least three experiments. ${ }^{*} \mathrm{p}<0.05,{ }^{* *} \mathrm{p}<0.01$. 